\title{
热液管状蠕虫的早期矿化机制及微生物 在矿化过程中的作用
}

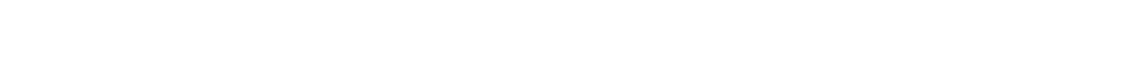 \\ (1) 中国科学院广州地球化学研究所, 极端环境地质地球化学重点实验室, 广州 510640; (2) 同济大学海洋地质国家重点实验室, \\ 上海 200092. E-mali: xtpeng@gig.ac.cn)
}

\begin{abstract}
摘要 热液喷口动物群是现代海底热液体系的重要组成部分, 它们依赖于热液无机环境生存, 与无机 环境之间存在着密切的相互作用, 并可参与现代热液点的成矿过程. 热液喷口动物群(特别是 Vestimentiferan和Polychaete管状蠕虫)矿化后的产物常以化石的形式保存于各时代的地质体中. 开展热 液大型动物的早期矿化过程研究, 对于理解热液环境中矿物与生物的相互作用以及地质化石的形成和 保存机制具有重要的意义. 以胡安. 德富卡洋脊热液场中采集的管状蠕虫Vestimentiferan Ridgeia piscesae为对象, 对它的早期生物矿化特征和机制进行了研究. 研究表明, 大量的丝状微生物不均匀地 分布在Ridgeia piscesae 管状蠕虫的内壁表面和壁内空隙层中, 并在一些部位形成微生物薄层. 微生物细 胞表面和降解后的产物在管状蠕虫矿化早期起着重要的作用. 在矿化程度较低的管状蠕虫管壁, 普遍 发现有半透明的含硫有机质薄层和球粒状颗粒硫的存在. 这种含硫有机薄层的降解产物在管状蠕虫早 期矿化过程中的作用可能同样不容忽视. 微区化学分析表明, 管状蠕虫管壁对成矿元素的富集具有选 择性, 主要从周围热液环境中富集 $\mathrm{Fe}, \mathrm{P}, \mathrm{Ca}$ 和 $\mathrm{Si}$ 等元素, $\mathrm{Fe}$ 与 $\mathrm{P}, \mathrm{Ca}$ 和 $\mathrm{Si}$ 等元素具有共变关系. 由于 $\mathrm{S}$ 主要 来源于管状蠕虫组织体中共生微生物对 $\mathrm{H}_{2} \mathrm{~S}$ 的生物氧化的作用, 它可作为研究管状蠕虫管壁矿化过程 的一种很好的生物标志物. 根据不同矿化程度管状蠕虫的矿化特征, 提出管状蠕虫的早期矿化过程主 要受微生物诱导生物矿化作用和管壁降解生物矿化作用控制.
\end{abstract}

关键词 生物矿化 管状蠕虫 热液 微生物 Ridgeia piscesae

作为一种“复杂的生物地质体”, 现代海底热液体 系中各种有机和无机组成单元是通过多种生化控制和 反馈作用相互联系起来的 ${ }^{[1 \sim 3]}$. 已有的证据表明, 喷口 生物群能够影响现代热液点的成矿过程 ${ }^{[4]}$. 例如, 生 长在动物和硫化物矿物表面的微生物席能作为多金 属壳层形成的场所 ${ }^{[5 \sim 7]}$; Alvinellid polychaetes 被认为 可以改变黑烟图形貌、孔隙度和矿物组成 ${ }^{[8,9]}$. 同样 的矿化过程亦可导致了喷口动物壳和有机质管体被 矿物所取代, 并随后加入到硫化物烟图的生长过程 中. 例如, 东太平洋海隆的Alvinellid polychaete管体 被硅质层和黄铁矿所取代 ${ }^{[8]}$; 胡安. 德富卡洋脊 Ridgeia piscesae管体被重晶石、无定形硅和晚期的Fe, $\mathrm{Zn}$ 和 Cu硫化物所取代 ${ }^{[10]}$; 中印度洋脊腹足动物骨片 被Fe硫化物所取代 ${ }^{[11]}$; 在Manus盆地中, 多腹足类软 体动物Ifremeria nautilei被富 Zn硫化物所取代 ${ }^{[12]}$. 开 展现代海底热液生物的矿化过程研究, 对于深入理
解现代热液成矿机制，揭示热液无机环境与有机生 物之间的相互作用具有十分重要的意义.

至今为止，已在几十处古代硫化物堆积体中发 现了热液喷口生物化石. 这些化石主要为 Vestimentiferan、多毛目环节动和其他一些未被识别 的管状蠕虫. 化石形成的地质年代跨度从志留纪到 始新世 ${ }^{[8,13 \sim 15]}$. 一些学者认为, 喷口生物的矿化过程是 快速发生的, 但准确的机制至今还不清楚 ${ }^{[13 \sim 15]}$. Cook和Stakes ${ }^{[10]}$ 曾研究了现代热液地质体中晚期硫 酸盐和硫化物矿物对喷口管状蠕虫的取代. Maginn等 人 [16]研究了现代热液场中多毛目环节动物 Alvinella pompejana的硫化物矿化过程. 多名学者已认识到, 微 生物可能在喷口大型生物的矿化过程中起重要作用 [17]. 然而, 目前对喷口大型生物的早期矿化过程知之 甚少, 特别是对生物壳壁的氧化物矿化过程研究极 少, 而这一过程对于理解古代硫化物堆积体中生物 
化石的形成同样十分重要.

本文以采自东北太平洋胡安. 德富卡洋脊 Main Endeavour 热液场的大型动物 vestimentiferan Ridgeia piscesae 为研究对象, 对它的早期生物矿化特征、生 物矿化机制及微生物在其中所起的作用做一探讨.

\section{1 材料与方法}

\section{1 样品采集}

2005 年 8 月 13 至 9 月 3 日, 中美首次在东北太 平洋胡安. 德富卡(JDF)洋脊开展了由Atlantis科考船和 Alvin深潜器执行的联合深潜航次. 本文研究所用 Vestimentiferan Ridgeia piscesae样品为此航次 4135 次下潜在Main Endeavour热液场扩散流环境中采集 的大型生物样品(图 1). Ridgeia piscesae 是JDF洋脊热 液场中独有的管状蠕虫种, 个体与其他洋脊管状蠕 虫相比较小, 与硫氧化化能自养细菌具有体内共生 关系 [18]. 管状蠕虫最外层为管壁, 是唯一可以化石 的形式保存在地质体中的身体部位, 由管状蠕虫内 部器官分泌物形成 ${ }^{[19]}$. Ridgeia piscesae样品由Alvin 深潜器机器手采集后, 被放置于生物箱中. 样品返回甲 板后, 立即分别用 $4 \%$ 的戊二醛固定和 $8 \%$ 的甲醛样品 固定, $2^{\circ} \mathrm{C}$ 保存. 挑选代表不同矿化程度的样品进行透 射电子显微镜、扫描电子显微镜、电子探针和背散射 显微分析. 各样品描述如下: $\mathrm{T} 1$ 为管壁样品, 外壁表面 灰白色; $\mathrm{T} 2$ 为管壁样品, 外壁表面红褐色; $\mathrm{T} 3$ 为管壁

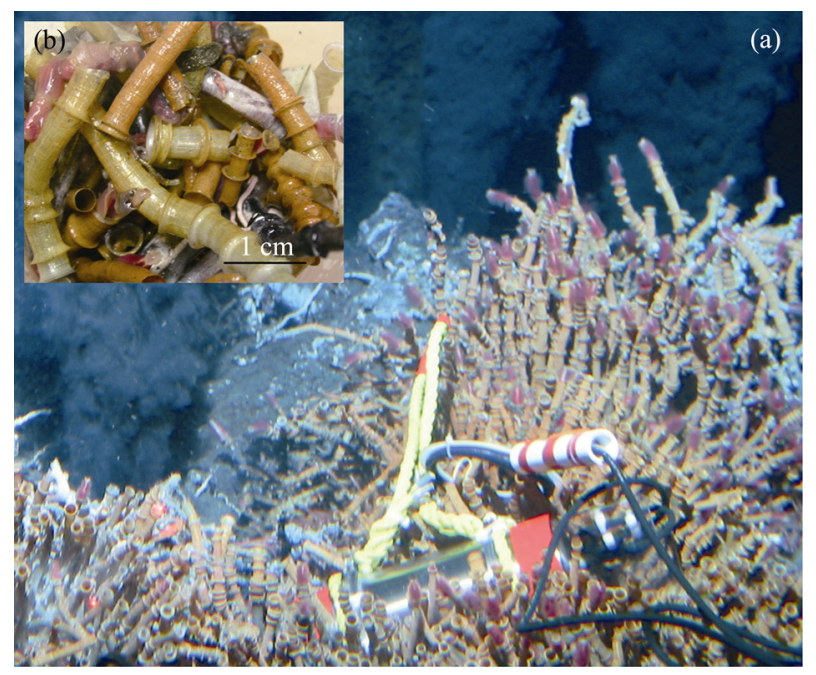

图 1

(a) Main Endeavour 热液场中的管状蠕虫 Ridgeia piscesae 和附近的活 动热液喷口，拍摄于中美联合深潜航次 4135 次下潜; (b) 采集到的部 分管状蠕虫样品，管状蠕虫表面颜色的差异，反映了不同个体之间矿 化程度的不同
样品, 外壁表面黄褐色; T4 为管体腔内的黄褐色组织 填充物. 矿物学分析表明, 管壁内主要含铁氧化物和 单质硫矿物.

\section{2 分析方法}

应用可检测活体、潮湿样品的荷兰 FEI Quant 400 型环境扫描电子显微镜, 采用低真空模式, 直接对不 经表面处理(不喷渡)的管状蠕虫样品进行观察, 以避 免前处理过程对管状蠕虫样品的破坏和可能对能谱 分析产生的影响. 扫描电子显微镜的工作条件: 加速 电压 $20 \mathrm{kV}$, 最大发射电流 $300 \mu \mathrm{A}$. 应用 EDAX 公司 Genesis 型能谱仪对管状蠕虫表面微区化学成分进行 分析. 能谱分析探头分辨率优于 $129 \mathrm{eV}$, 峰背比优于 20000: 1, 检测元素范围 Be4-U92, 最大计数率 500000 cps.

用荷兰 FEI Tecnai 12 型透射电子显微镜对管状 蠕虫超薄切片样品进行观察, 加速电压 $100 \mathrm{kV}$. 采用 英国牛津 INCA Energy TEM X 射线能谱仪, 以无标 样定量方式, 分析微区化学成分. 透射电子显微镜样 品前处理过程如下: 将管状蠕虫样品分切成 $0.15 \mathrm{~cm}$ $\times 0.45 \mathrm{~cm}$ 的若干长条形小块, $2 \%$ 戊二醛 $4{ }^{\circ} \mathrm{C}$ 固定 $2 \mathrm{~h}$, PBS 溶液洗 3 次, 每次 $10 \mathrm{~min} .4^{\circ} \mathrm{C}$ 下浸泡于 $1 \%$ 锇酸 溶液中 $2 \mathrm{~h}$, 用 $10 \%, 20 \%, 30 \%, 40 \%, 50 \%, 60 \%, 70 \%$, $80 \%, 90 \%$ 和 $100 \%$ 的系列乙醇梯度脱水各 $10 \mathrm{~min}$. 经 环氧丙烷过渡到环氧树脂渗透包埋, 超薄切片, 醋酸 铀-柠檬酸铅双染.

应用日本 JEOL JXA-8100 型电子探针仪(EPMA), 对管状蠕虫管壁元素组成进行定性、定量及背散射图 像分析. EPMA 的波长范围为 $0.087 \sim 9.3 \mathrm{~nm}$, 定量分 析的检测极限 $100 \mu \mathrm{g} / \mathrm{g}$, 二次电子像分辨率为 $6 \mathrm{~nm}$, 背散射电子像分辨率为 $20 \mathrm{~nm}$, 放大倍数为 40 300000 倍. 分析电压为 $20 \mathrm{kV}$, 探针电流为 $2 \times 10^{-8} \mathrm{~A}$, 束斑 尺寸为 $1 \mu \mathrm{m}$.

\section{2 结果与讨论}

\section{1 微生物在管壁上的分布}

扫描电子显微镜与透射电子显微镜观察表明, 微生物主要不均匀地分布在 Ridgeia piscesae 管状蠕 虫的内壁表面和壁内空隙层中, 并在一些部位形成 的微生物薄层(图 2). 管状蠕虫外壁表面的微生物明 显少于内壁表面. 微生物内壁表面和壁内空隙层中 的微生物以丝状微生物为主(图 2, 3). 这些丝状微生 物主要分为两类, 23 类直径约 $0.1 \mu \mathrm{m}$, 另一类直径约 


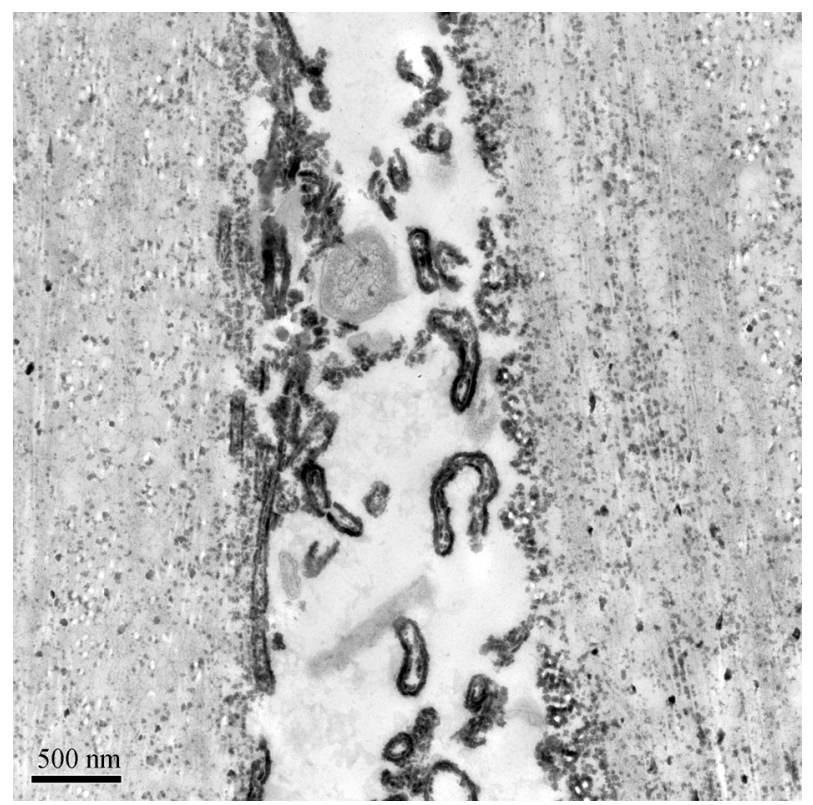

图 2 管状蠕虫透射电子显微镜(TEM)图 壁内空隙层表面附着大量丝状微生物, 并形成微生物薄层. 一些微生 物已经降解, 形成纳米级小颗粒分布于空隙层表面和管壁内

$0.3 \sim 0.5 \mu \mathrm{m}$. 在第一类丝状微生物外表面常发育鞘层. 这些微生物在形态上与管状蠕虫营养体内的共生微生 物具有明显区别，因此并非是在管状蠕虫新陈代谢过 程中起重要作用的共生微生物. 不同微生物个体之 间矿化程度存在一定的差异, 有的微生物表面发生了 部分矿化, 而有的微生物已被完全矿化. 在内壁表面和 壁内间隙层中观察到大量微生物发生了降解, 降解后 形成的纳米级小颗粒黏附在内壁表面和分布于管壁内. 显微观察发现，管壁内部同样存在有与内壁表面
相似的丝状微生物. Gaill和Hunt ${ }^{[20,21]}$ 认为, 它们是随 着有机分子在内壁表面的累积而逐渐进入壁内的. 这 些微生物在被掩埋后, 依然保持着形态上的完整性.

\section{2 内外壁表面的微形貌和元素分布特征}

不同管状蠕虫个体之间外壁表面微形貌的差异, 反映了个体之间矿化程度的不同. 扫描电子显微镜 观察发现，在矿化程度很低的样品(如灰白色 T1 样品) 上, 外壁表面通常较光滑, 内壁和外壁表面普遍存在 着半透明的有机薄层, 并可发现球粒状颗粒硫的存 在(图 4). 多层含硫薄层相互叠加, 形成微叠层结构. 而在矿化程度较高的样品(如深褐色 $\mathrm{T} 2$ 样品)上, 外 表面微形貌粗䊁, 并有开裂现象, 可观察到许多直径 为数微米至数十微米的铁氧化物聚集体形成于其外 表面上(图 5). 在矿化程度较高的管壁外表面没有观 察到含硫有机薄层的存在, 仅在内壁表面局部区域 发现了含硫有机薄层. 含硫有机薄层在矿化程度较 高的管壁中的缺失表明，管壁的矿化过程与含硫有 机薄层的降解过程是一对相关过程. 前人研究表明, 管状蠕虫管壁中的有机质包括壳多糖(一种含氮多聚 糖)和蛋白质等 $[20,21]$. 这些有机质降解产物对成矿元 素的吸附可能极大地促发了管壁的矿化 ${ }^{[22,23]}$.

能谱分析表明，管状蠕虫对热液元素的富集具 有一定的选择性, 主要富集 $\mathrm{Fe}, \mathrm{P}, \mathrm{Ca}$ 和 $\mathrm{Si}$ 等元素, 但 不同个体因矿化程度的不同对上述元素富集程度具 有较明显的差异性.对管状蠕虫内、外壁表面微区分析 发现，管状蠕虫外壁表面的矿化程度明显高于内壁表 面. 例如, $\mathrm{T} 3$ 样品外壁表面以富 $\mathrm{Fe}, \mathrm{P}, \mathrm{Ca}$ 和 $\mathrm{Si}$ 为
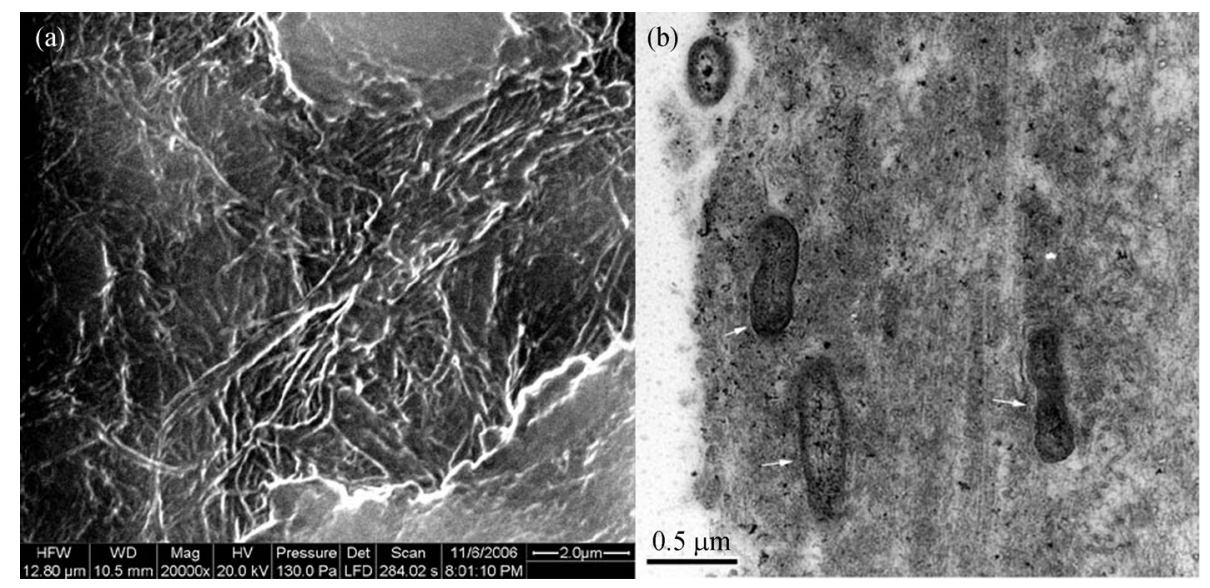

图 3

(a) 管状蠕虫内壁表面扫描电子显微镜(SEM)图, 可见大量丝状微生物聚集于半透明的含硫有机层内; (b) 管壁 TEM 图, 箭头所指为管壁内微生物 

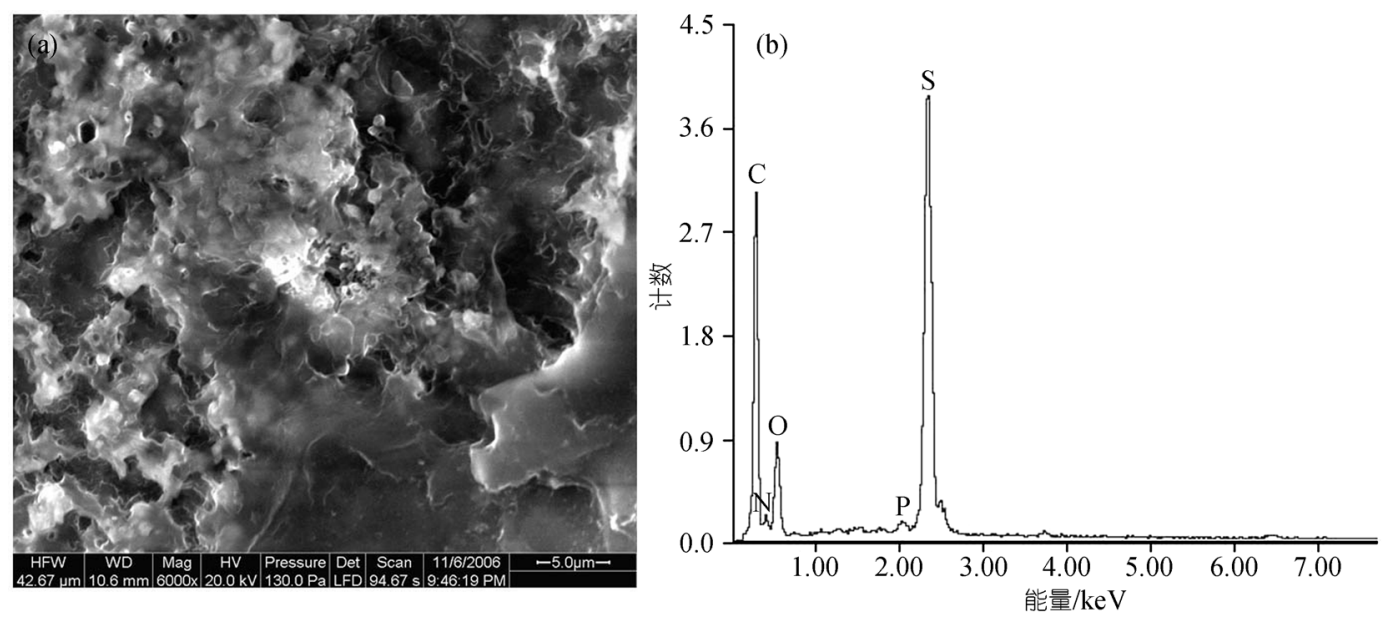

图 4

(a) T1 样品内壁表面的硫薄层和球粒状硫颗粒; (b) 含硫有机薄层能谱分析
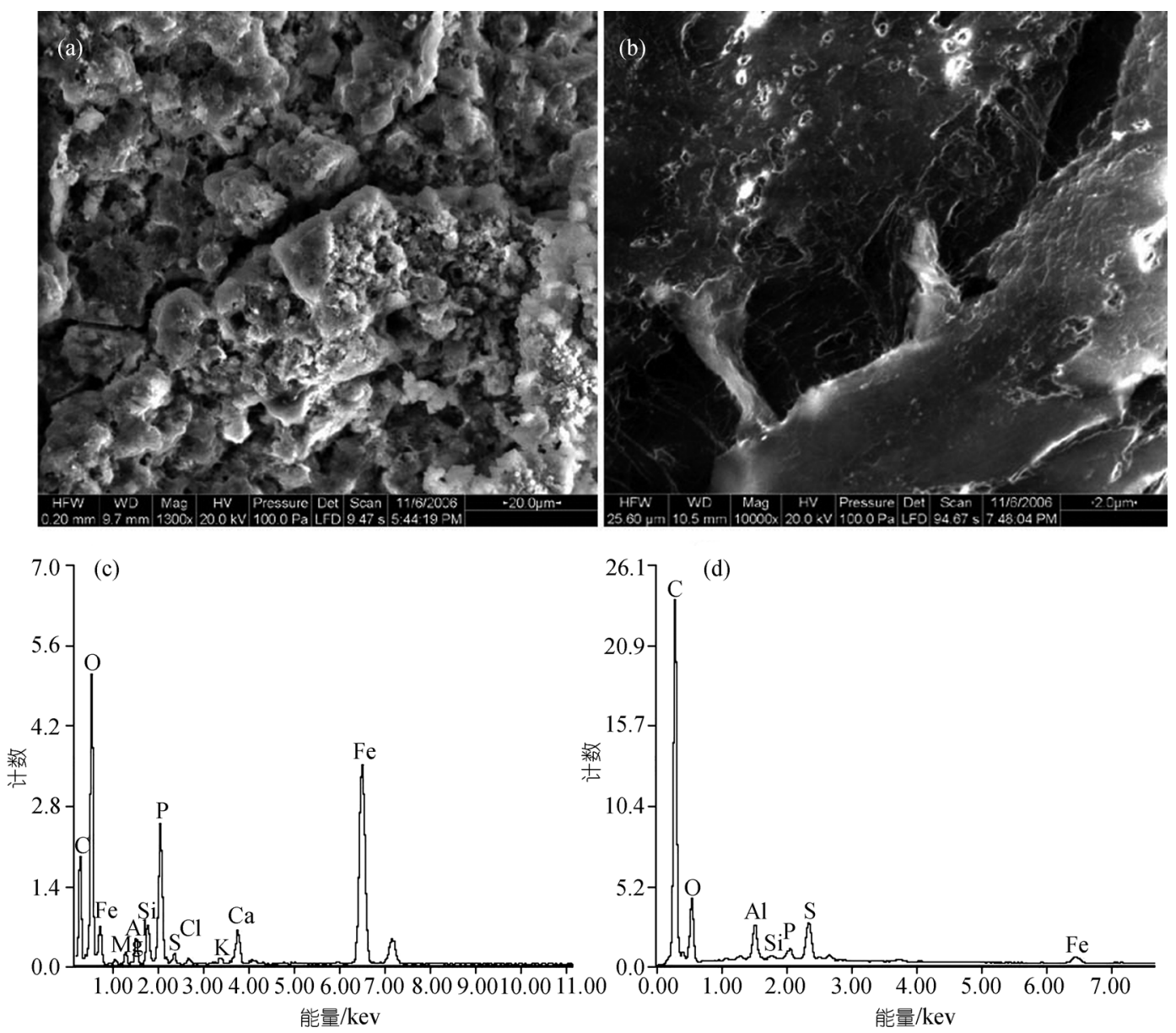

图 5 不同矿化程度管状蠕虫外壁表面微形貌和元素分布的差异

(a) T2 样品外壁表面局部粗粘开裂的微形貌, 其上存在大量铁氧化物聚集体, 矿化程度高; (b) T3 样品外壁相对较光滑, 并有观察到半 透明的含硫有机薄层; (c) T2 样品外壁表面能谱分析, Fe, P 和 Ca 等元素含量高; (d) T3 样品外壁表面能谱分析, C 和 S 等元素含量相对 https://engine.sc较高, 并含 $\mathrm{Fe}, \mathrm{Al}, \mathrm{Si}$ 和 $\mathrm{P}$ 等元素 $2007-52-23-2759$ 
特征, 内壁表面 $\mathrm{Fe}, \mathrm{P}, \mathrm{Ca}$ 和 $\mathrm{Si}$ 等元素含量显著减少, 但 S 含量增加; T2 样品内外壁表面的能谱分析表现出 了同样的趋势, 外壁表面 $\mathrm{Fe}$ 高 $\mathrm{S}$ 低, 而内壁表面 $\mathrm{S}$ 高 $\mathrm{Fe}$ 低; $\mathrm{T} 1$ 样品的矿化程度最低, 在其内外壁表面, 能谱分析未能检出 $\mathrm{Fe}, \mathrm{P}$ 和 $\mathrm{Ca}$ 等元素, 但 $\mathrm{S}$ 的分布趋 势与 $\mathrm{T} 2, \mathrm{~T} 3$ 样品相同, 呈现出外壁 $\mathrm{S}$ 低而内壁 $\mathrm{S}$ 高.

\section{3 管壁剖面的化学特征和元素来源分析}

将管状蠕虫制成探针片后，用 EPMA 对管壁剖 面和腔内组织填充物剖面的元素分布特征和趋势进 行分析. 表 1 3 显示了 $\mathrm{Fe}, \mathrm{P}, \mathrm{S}, \mathrm{Ca}, \mathrm{Si}, \mathrm{Cu}, \mathrm{Pb}$ 和 $\mathrm{Zn}$ 等元素在 $\mathrm{T} 1, \mathrm{~T} 2$ 和 $\mathrm{T} 4$ 样品管壁剖面和组织剖面中的 含量. 可以发现, 腔内组织填充物样品(T4 样品)的矿 化程度最低, 主要成分为 $\mathrm{S}$ 和有机质. 在 $\mathrm{T} 2$ 管壁样 品中, 总无机元素含量可大于 $40 \%$. 在管壁剖面上, $\mathrm{Fe}$ 含量与 $\mathrm{P}, \mathrm{Ca}$ 等元素含量具有共变关系, 呈正相关, 而 $\mathrm{S}$ 的含量则与 $\mathrm{Fe}, \mathrm{P}, \mathrm{Ca}$ 基本呈现负相关. 元素面分 析同样显示了相似的结果: 在 $\mathrm{Fe}$ 含量高的区域, $\mathrm{P}$ 和 $\mathrm{Ca}$ 的含量高, 而 $\mathrm{S}$ 含量相对较低(特别在 $\mathrm{T} 2$ 样品中). 前人研究表明, 在生物体中, $\mathrm{P}$ 与有机质密切联系, 可作为一种生物标志物指示地质体中生命活动的存

表 $1 \mathrm{~T} 1$ 管壁剖面化学成分电子探针分析 ${ }^{\mathrm{a})}$

\begin{tabular}{|c|c|c|c|c|c|c|c|c|}
\hline $\begin{array}{c}\text { 元素 } \\
(\%)\end{array}$ & $\begin{array}{c}\text { 点 } 1 \\
\text { (B) }\end{array}$ & 点 2 & 点 3 & 点 4 & 点 5 & 点 6 & 点 7 & $\begin{array}{l}\text { 点 } 8 \\
\text { (A) }\end{array}$ \\
\hline $\mathrm{Ca}$ & 0.368 & 0.355 & 1.124 & 1.193 & 0.503 & 0.259 & 0.188 & 0.136 \\
\hline $\mathrm{O}$ & 3.372 & 3.192 & 4.772 & 3.908 & 3.488 & 3.096 & 2.903 & 3.011 \\
\hline $\mathrm{Si}$ & 0.137 & 0.123 & 0.493 & 0.621 & 0.237 & 0.103 & 0.069 & 0.058 \\
\hline $\mathrm{P}$ & 0.568 & 0.666 & 1.788 & 1.986 & 0.804 & 0.457 & 0.362 & 0.267 \\
\hline $\mathrm{Fe}$ & 1.405 & 1.19 & 5.477 & 6.665 & 2.237 & 0.986 & 0.758 & 0.598 \\
\hline S & 3.31 & 4.517 & 2.904 & 2.851 & 3.926 & 3.44 & 4.117 & 4.979 \\
\hline $\mathrm{Cu}$ & 0.006 & 0.021 & 0.014 & 0.095 & 0.057 & 0.005 & 0.005 & - \\
\hline $\mathrm{Pb}$ & 0.029 & - & 0.02 & - & 0.003 & 0.006 & 0.001 & 0.003 \\
\hline $\mathrm{Zn}$ & 0.039 & 0.002 & 0.057 & 0.122 & 0.044 & 0.052 & - & 0.013 \\
\hline 总量 & 9.234 & 10.066 & 16.649 & 17.441 & 11.299 & 8.404 & 8.403 & 9.065 \\
\hline
\end{tabular}

表 2 T2 管壁剖面化学成分电子探针分析

\begin{tabular}{lcccccccc}
\hline $\begin{array}{l}\text { 元素 } \\
(\%)\end{array}$ & $\begin{array}{c}\text { 点 } 1 \\
(\mathrm{~A})\end{array}$ & 点 2 & 点 3 & 点 4 & 点 5 & 点 6 & 点 7 & $\begin{array}{c}\text { 点 } 8 \\
(\mathrm{~B})\end{array}$ \\
\hline $\mathrm{Ca}$ & 0.998 & 1.055 & 1.068 & 0.919 & 0.617 & 0.399 & 0.518 & 0.742 \\
$\mathrm{O}$ & 8.466 & 13.613 & 5.304 & 8.137 & 6.364 & 2.901 & 3.701 & 3.901 \\
$\mathrm{Si}$ & 2.807 & 3.657 & 2.544 & 1.654 & 0.775 & 0.139 & 0.303 & 0.322 \\
$\mathrm{P}$ & 3.026 & 3.591 & 2.589 & 1.721 & 0.874 & 0.329 & 0.512 & 0.645 \\
$\mathrm{Fe}$ & 29.111 & 26.668 & 20.04 & 11.248 & 5.536 & 1.019 & 2.25 & 3.058 \\
$\mathrm{~S}$ & 0.331 & 0.661 & 1.235 & 2.828 & 4.821 & 4.491 & 4.467 & 4.183 \\
$\mathrm{Cu}$ & 0.008 & 0.016 & 0.03 & - & 0.026 & 0.011 & 0.024 & 0.073 \\
$\mathrm{~Pb}$ & 0.028 & - & - & 0.013 & - & - & 0.012 & 0.024 \\
$\mathrm{Zn}$ & 0.059 & 0.011 & 0.03 & 0.007 & 0.061 & 0.045 & 0.073 & 0.094 \\
总量 & 44.834 & 49.272 & 32.84 & 26.527 & 19.074 & 9.334 & 11.86 & 13.042 \\
\hline
\end{tabular}

表 $3 \mathrm{~T} 4$ 组织填充物化学成分电子探针分析

\begin{tabular}{lccccccc}
\hline $\begin{array}{c}\text { 元素 } \\
(\%)\end{array}$ & $\begin{array}{c}\text { 点 } 1 \\
(\mathrm{~A})\end{array}$ & 点 2 & 点 3 & 点 4 & 点 5 & 点 6 & $\begin{array}{c}\text { 点 } 7 \\
(\mathrm{~B})\end{array}$ \\
\hline $\mathrm{Ca}$ & 0.171 & 0.129 & 0.139 & 0.141 & 0.138 & 0.275 & 0.766 \\
$\mathrm{O}$ & 3.296 & 8.302 & 6.468 & 4.693 & 2.555 & 3.209 & 5.036 \\
$\mathrm{Si}$ & 0.01 & 0.027 & 0.023 & 0.004 & 0.01 & 0.005 & 0.016 \\
$\mathrm{P}$ & 0.206 & 0.266 & 0.269 & 0.231 & 0.141 & 0.311 & 0.411 \\
$\mathrm{Fe}$ & 0.183 & 0.195 & 0.18 & 0.143 & 0.078 & 0.146 & 0.202 \\
$\mathrm{~S}$ & 3.824 & 4.892 & 5.332 & 8.1 & 7.062 & 8.722 & 8.521 \\
$\mathrm{Cu}$ & 0.044 & 0.057 & 0.045 & 0.042 & 0.013 & 0.038 & 0.047 \\
$\mathrm{~Pb}$ & 0.008 & - & 0.025 & - & - & - & - \\
$\mathrm{Zn}$ & 0.047 & 0.065 & 0.073 & 0.088 & 0.041 & 0.069 & 0.059 \\
总量 & 7.789 & 13.933 & 12.554 & 13.442 & 10.038 & 12.775 & 15.058 \\
\hline
\end{tabular}

在 ${ }^{[16]}$, 而本次研究表明, 在管状蠕虫管壁中, $\mathrm{S}$ 更适 合作为一种指示有机质含量高低的生物标志物. 电 子探针分析表明, 管壁内 $\mathrm{Fe}$ 主要以 $\mathrm{Fe}$ 氧化物的形式 存在, 未发现铁硫化物的存在.

电子背散射图像和元素面图像清晰地显示了管 壁和组织填充物横截面的矿化特征(图 6 8): (i ) 背 散射图像亮条带基本与管壁表面平行, 继承了管壁 分层结构的特点; (ii ) $\mathrm{Fe}, \mathrm{P}, \mathrm{Ca}$ 和 $\mathrm{Si}$ 等元素的高含量 条带分布基本与背散射图像亮条带位置一致, 反映 了亮条带区域即为管壁的早期矿化区域; (iii) 在矿 化程度较低的管壁样品中, 矿化作用优先在某些平 行于管壁的部位发生(如在 $\mathrm{T} 1$ 样品中); (iv) 在矿化程 度较高的样品中, 矿化作用从内到外总体有增强趋 势(尤其在 $\mathrm{T} 2$ 样品中); ( v ) S 的含量变化大体与 $\mathrm{Fe}, \mathrm{P}$, $\mathrm{Ca}$ 和 $\mathrm{Si}$ 等元素变化相反, 反映了两类元素不同的来 源, 后者来源于热液贡献; ( vi) 越靠近内壁 $\mathrm{S}$ 含量越 高, 特别是组织填充物中 $\mathrm{S}$ 的含量达到最大值, 表明 $\mathrm{S}$ 主要来自于管状蠕虫中共生微生物化能合成过程的

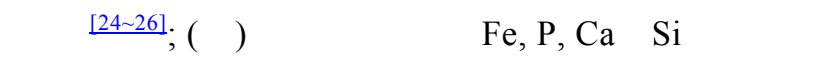
没有明显的富集趋势, 显示矿化作用在该部位并未 发生.

热液地质体和生物体中 $\mathrm{S}$ 的来源十分广泛, 既可 来自于海水中 $\mathrm{SO}_{4}^{2-}$ 还原, 亦可源自热液流体中 $\mathrm{H}_{2} \mathrm{~S}$,

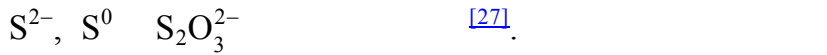
氧化菌在热液环境中各种硫形态相互转换过程中起 着重要作用. 在管状蠕虫中, 硫氧化共生菌对 $\mathrm{H}_{2} \mathrm{~S}$ 气 体的生物氧化作用是管体内 $S$ 的富集的主要原因 ${ }^{[24,25]}$. 生物学者已在管状蠕虫营养体内观察到球粒状元素 硫包裹体与原核细胞共存的现象, 是共生微生物参 与硫化氢氧化的有利证据 ${ }^{[25]}$. 这些共生微生物通过 氧化硫化氢产生ATP和通过生物合成途径固定无机 碳 (Calvin-Benson循环) 来完成化能合成自 
(a)

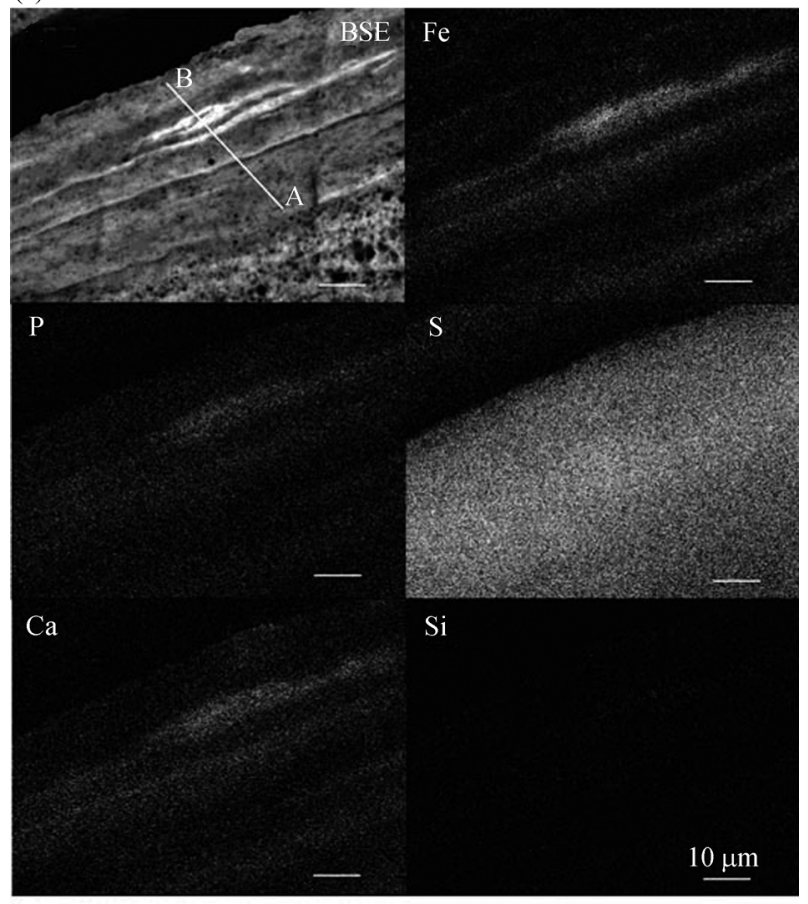

(b)

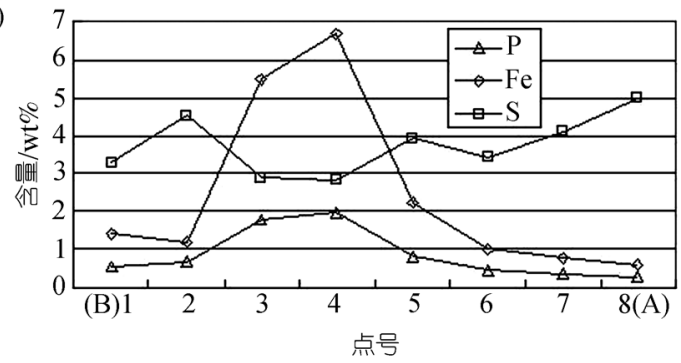

图 6

(a) T1 管壁剖面背散射图(BSE)与元素面( $\mathrm{Fe}, \mathrm{P}, \mathrm{S}, \mathrm{Ca}, \mathrm{Si}$ )图, 矿化作用 优先在某些平行于管壁的不连续层中发生; (b) T1 样品 A-B 剖面 $\mathrm{Fe}, \mathrm{P}$,

$\mathrm{S}$ 分布趋势, 三者具有共变关系. $\mathrm{wt} \%$ 为质量分数, 下同

养过程 ${ }^{[26]}$. 在硫氧化共生菌对热液 $\mathrm{H}_{2} \mathrm{~S}$ 气体的氧化过 程中, 元素硫是其中的重要产物之一. 本次研究在管 状蠕虫管壁和组织样品中观察到了球状硫颗粒的存在. 电子探针分析显示, $\mathrm{S}$ 在组织填充物中含量最高, 同样 表明管状蠕虫中硫来自于体内共生微生物的新陈代 谢贡献. 我们认为, $\mathrm{S}$ 的这种微生物来源, 使得它可以 作为管状蠕虫体内的一种生物标志物, 并在研究管 状蠕虫管壁的矿化中提供了重要的信息. 由于元素 硫的形成以后在矿化过程中还可进一步被氧 化成 $\mathrm{H}_{2} \mathrm{SO}_{4}^{-}$而从管体中散失，因此， $\mathrm{S}$ 在管壁中的含 量高低一方面可以反映了有机质的高低，另一方面 亦可反映管状蠕虫矿化程度的高低. 也就是说, $S$ 高 时指示矿化程度低，而 $\mathrm{S}$ 低时指示矿化程度高. 在管 壁剖面上, $\mathrm{S}$ 的分布趋势与 $\mathrm{Fe}, \mathrm{P}, \mathrm{Ca}$ 基本相反, 较好地 (a)
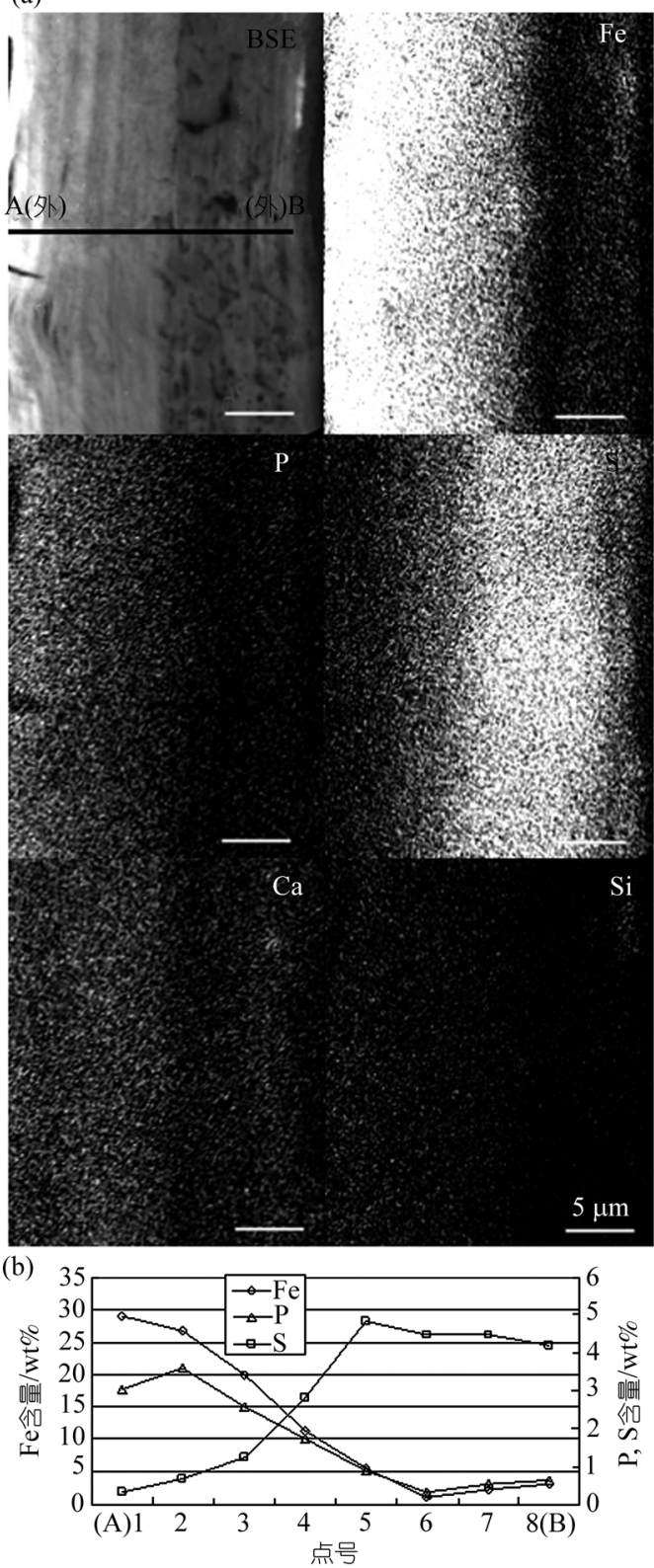

图 7

(a) T2 管壁剖面背散射图(BSE)与元素面( $\mathrm{Fe}, \mathrm{P}, \mathrm{S}, \mathrm{Ca}, \mathrm{Si}$ )图, 矿化作用 由内至外明显增强; (b) T2 样品 A-B 剖面 $\mathrm{Fe}, \mathrm{P}, \mathrm{S}$ 分布趋势, $\mathrm{S}$ 含量在 剖面上的变化与 $\mathrm{Fe}, \mathrm{P}$ 的变化相反

指示了管壁的矿化程度.

由于 $\mathrm{P}$ 是生物体内重要的化学元素，它常被用于 作为指示地质体中生命体存在的标志 ${ }^{[16]}$. 在Ridgeia piscesae组织体中, $\mathrm{P}$ 的含量在 0.14 0.41 之间变化, 与 其他一些热液生物体(如Alvinellid polychaetes)中P含量 相近. 但在管壁中, P的含量远高于组织体, 约为 10 倍, 与 $\mathrm{Fe}, \mathrm{Ca}, \mathrm{Si}$ 等元素具有共变关系, 并呈现出由外壁向 内壁降低的趋势，这表明管壁中的 $\mathrm{P}$ 与 $\mathrm{Fe}, \mathrm{Ca}$ 


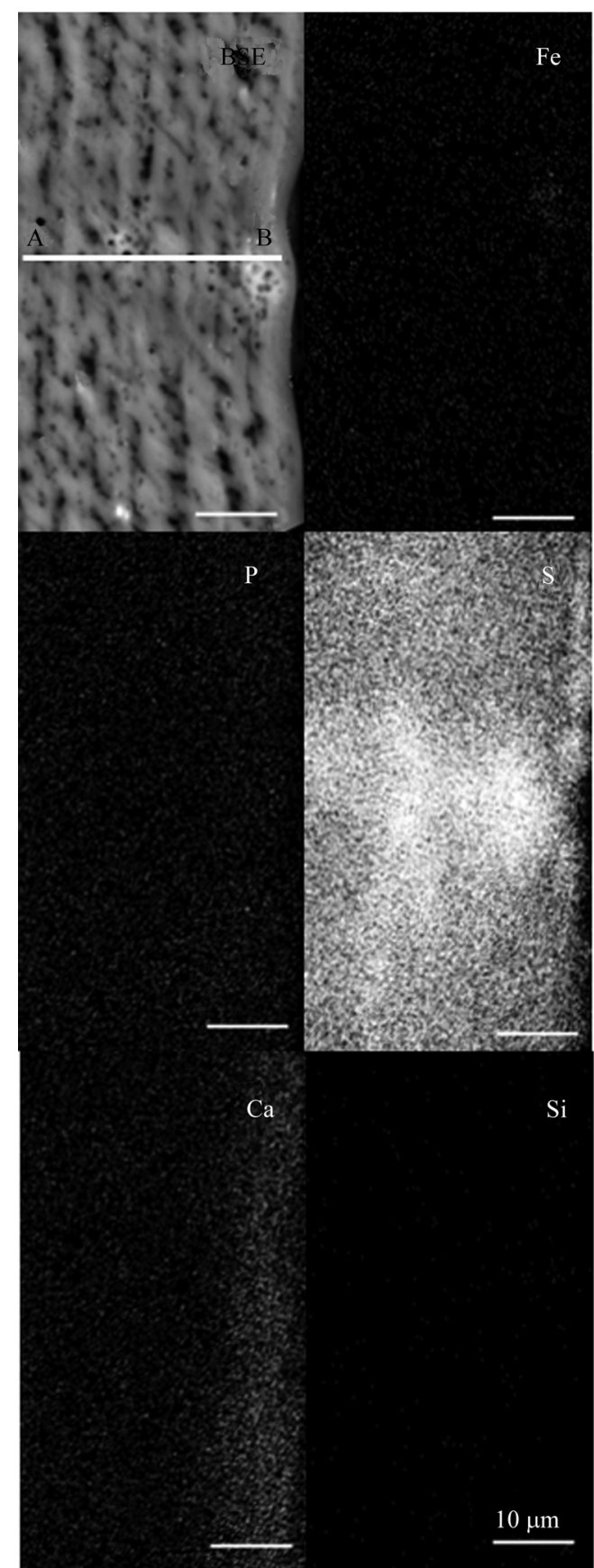

图 $8 \mathrm{~T} 4$ 样品背散射图(BSE)与元素面 $(\mathrm{Fe}, \mathrm{P}, \mathrm{S})$ 图 $\mathrm{Fe}, \mathrm{P}$ 基本未富集; $\mathrm{S}$ 含量高, 并在某些部位形成明显的亮点

和 $\mathrm{Si}$ 一样, 主要来源于外部, 是生物矿化过程中从周 围热液环境中进入管壁内部的. 由于来自生物体内 部的 $\mathrm{P}$ 占总 $\mathrm{P}$ 比例低, 因此, 在研究 Ridgeia piscesae 管状蠕虫管壁矿化时, $\mathrm{P}$ 已不适合作为一种生物标志 物来指示有机质含量的高低.

\section{4 管壁对微生物的捕获}

多种显微观察发现，管状蠕虫的管壁表面和内
部存在着众多的微生物. 微生物在管壁内的存在, 为 管壁内成矿元素的富集提供了极好的微环境, 使这 些微生物富集区域成为管壁中优先发生矿化作用的 场所 ${ }^{[7,16]}$. 通常, 矿物在微生物细胞的表面沉淀可用 两种机制进行解释: ( i ) 微生物细胞表面直接从流 体中捕获矿物颗粒和( ii )因周围环境离子浓度升高, 成矿离子在细胞表面与其他离子结合而沉淀 ${ }^{[28,29]}$. 我们认为后一种机制最有可能在管壁内发生. 在后 一种情况下, 微生物生长于管壁表面或被管壁产生 的蛋白质层所捕获. 由于微生物连续与通过管壁的 流体接触, 当其在被包埋在管壁内之前, 微生物可将 成矿离子吸附在细胞膜上. 一旦微生物细胞被管壁 掩埋, 并开始降解, 细胞膜内存在的离子将进一步诱 导溶液中离子成核作用的发生 $[16,30]$. 微生物降解产 物(如各种阴离子和阳离子有机功能团)的形成, 将更 有效地提高微生物层的生物矿化潜力. 我们观察到 许多微生物和微生物降解形成的小颗粒吸附于管状 蠕虫管壁表面和内部, 矿化作用已在这些微生物表 面和降解形成的小颗粒上发生, 它们的矿化程度明 显高于周围管壁(图 9)。管壁表面和内部微生物及降 解产物的存在, 在管状蠕虫矿化的早期阶段对成矿 元素的富集起着重要的作用, 并可能是导致在矿化 程度较低的样品(如 $\mathrm{T} 1$ 样品)中矿化作用优先在一些 平行于管壁的不连续层发生的主要原因.

\section{5 管壁矿化的可能机制}

由于微生物主要被发现在矿化程度较低的管状 蠕虫内壁表面和壁内间隙空间内聚集, 并且在微生 物的表面明显存在着矿化的现象, 据此我们认为管 状蠕虫管壁的矿化过程最初是从这些微生物上开始. 微生物细胞外表面和胞外物质所具备的对 $\mathrm{Fe}, \mathrm{S}, \mathrm{Ca}$ 和 $\mathrm{Si}$ 等多种成矿元素的捕获能力, 使早期发生于细 胞表面的矿化得以发生. 微生物在被掩埋后, 一部分 微生物的细胞组分开始降解, 早期形成的矿物或矿 物先驱物可作为继续矿化的矿化点(图 9(d)). 这种微 生物诱导生物矿化方式常出现于矿化程度较低的样 品中(如 $\mathrm{T} 1$ 样品), 以矿化作用优先在微生物富集层 中发生, 所形成的矿化区域为平行于有机层的不连 续层为特点(图 6). 微生物诱导矿化方式使管壁矿化 部分继承了管壁某些结构(如微生物富集层)的特征, 从而使管壁上原有的一些结构特征得以保存.

能谱和电子探针分析表明，管壁有机质的降解作 用可以同样可能是导致管壁矿化的重要原因. 随着 


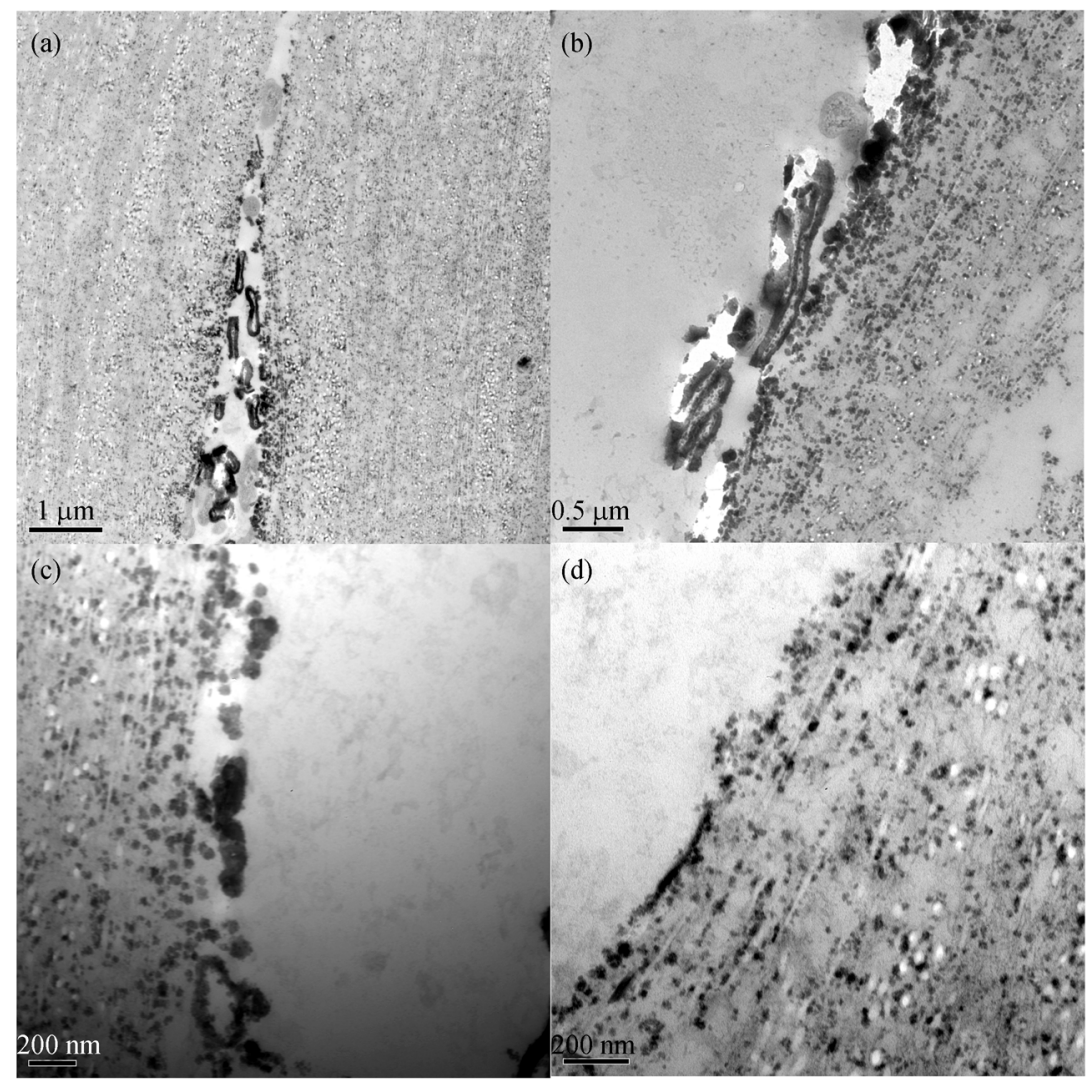

图 9 微生物及降解产物在管壁上的分布(TEM 照片)

(a) 微生物富集于管壁空隙层中; (b) 微生物在管壁内表面存在, 其中部分微生物已降解和矿化; (c) 管壁空隙层中已矿化的微生物和 降解形成的小颗粒; (d) 降解后形成的小颗粒随管壁的增厚进入壁内, 并基本平行于管壁内表面分布

管壁的增厚，管状蠕虫外壁有机质(特别是含硫的有 机质薄层)的生物活性会逐渐散失, 其中的蛋白质和 壳多糖等有机物将发生降解 [20,21]. 降解所形成产物, 对成矿离子具有重要的吸附, 有效地提高了管壁的 矿化潜力, 促进了矿化过程在管壁内的发生.由于外 壁最先形成, 其降解作用也较先发生, 加之管体外部 与热液流体直接接触, 故管体外表面矿化速度最快, 管壁矿化程度由外到内呈现出一种递减的趋势(如 $\mathrm{T} 2$ 样品, 见图 7). 这种生物矿化方式同样可能使管壁中 原有的分层结构得以保存.

\section{3 结论}

在热液管状蠕虫 Ridgeia piscesae 的内壁表面和 壁内空隙层中发现了大量的丝状微生物. 这些微生 物的表面及降解产物能有效地吸附周围环境中的成 矿离子, 使管壁的早期矿化过程优先在微生物聚集 层中发生. 在矿化程度较低的管状蠕虫内壁和外壁 表面, 发现了半透明的含硫有机质薄层和球粒状硫
颗粒的存在. 管状蠕虫管壁中的 $\mathrm{Fe}, \mathrm{P}, \mathrm{Ca}$ 和 $\mathrm{Si}$ 等元 素主要来自于周围热液环境, 是管壁对这些元素生 物富集作用的结果. 管壁中 $\mathrm{S}$ 则主要来自于体内共生 微生物的新陈代谢贡献. $\mathrm{S}$ 的这种生物来源, 使得它 可以作为管状蠕虫体内的一种生物标志物, 并在研 究管状蠕虫管壁的矿化中提供了重要的信息. 管状 蠕虫管壁的早期矿化过程可能主要受微生物诱导生 物矿化和管体降解生物矿化两种作用的控制. 前者 以矿化作用优先在微生物富集层中发生, 所形成的 矿化区域为平行于管壁有机层的不连续层为特点, 后者以管壁矿化程度由外到内总体呈现递减趋势为 特征. 热液喷口大型生物管状蠕虫的这种生物矿化 机制使得管壁的结构特征能够在化石中得以保留.

$$
\text { 参考文献 }
$$

1 Holden J F, Adams M W W. Microbe-metal interactions in marine hydrothermal environments. Curr Opin Chem Biol, 2003, 7: 1603165[DOH] 07-52-23-2759 
2 Reysenbach A L, Shock E S. Merging genomes with geochemistry in hydrothermal ecosystems. Science, 2002, 296: 1077-1082[DOI]

3 Edwards K J, Bach W, McCollom T M. Geomicrobiology in oceanography: Microbe-mineral interactions at and below the seafloor. Trends Microbiol, 2005, 13 (9): 449-456[DOI]

4 Juniper S K, Jonassan I R, Tunnicliffe V, et al. Influence of a tube building polycheate on hydrothermal chimney mineralisation. Geology, 1992, 20: 895-898[DOI]

5 Jannasch H W, Wirsen C O. Morphological survey of microbial mats near deep-sea thermal vents. Appl Environ Microbiol, 1981, 41: $528-538$

6 Emerson D, Moyer C I. Neutrophilic Fe-oxidizing bacteria are abundant at the Loihi Seamount hydrothermal vents and play a major role in Fe oxide deposition. Appl Environ Microbiol, 2002, 68: 3085-3093[DOI]

7 Tunnicliffe V, Fontaine A R. Faunal composition and organic surface encrustations at hydrothermal vents on the southern Juan de Fuca Ridge. J Geophys Res, 1987, 92: 11303-11314[DOI]

8 Haymon R M, Koski R A, Sinclair C. Fossils of hydrothermal vent worms from Cretaceous sulphide ores of the Samail ophiolite, Oman. Science, 1984, 223: 1407-1409[DOI]

9 Juniper S K, Sarrazin J. Interaction of vent biota and hydrothermal deposits: Present evidence and future experimentation. In: Humphris S E, Zierenberg R A, Mullineaux L S, et al, eds. Seafloor Hydrothermal Systems: Physical, Chemical, Biological, and Geological Interactions. Am Geophys U Monogr, 1995, 91: 178-193

10 Cook T L, Stakes D S. Biogeological mineralization in deep-sea hydrothermal deposits. Science, 1995, 267: 1975-1979[DOI]

11 Suzuki Y, Kopp R E, Kogure T, et al. Sclerite formation in the hydrothermal-vent "scaly-foot" gastropod: Possible control of iron sulfide biomineralization by the animal. Earth Planet Sci Lett, 2006, 242: 39-50[DOI]

12 Tufar W. Modern hydrothermal activity, formation of complex massive sulfide deposits and associated vent communities in the Manus back-arc basin (Bismarck Sea, Papua New Guinea). Mitt Osterr Geol Desaushaft, 1990, 82: 183-210

13 Little C T S, Herrington R J, Maslennikov V V, et al. Silurian hydrothermal vent community from the Southern Urals of Russia. Nature, 1997, 385: 146-148[DOI]

14 Little C T S, Cann J R, Herrington R J, et al. Late Cretaceous hydrothermal vent communities from the Troodos Ophiolite, Cyprus. Geology, 1999, 27: 1027-1030[DOI]

15 Little C T S, Herrington R J, Haymon R M, et al. Early Jurassic hydrothermal vent community from the Franciscan Complex, San
Rafael Mountains, California. Geology, 1999, 27: 167-170[DOI]

16 Maginn E J, Little C T S, Herrington R J, et al. Sulphide mineralisation in the deep sea hydrothermal vent polychaete, Alvinella pompejana: Implications for fossil preservation. Mar Geol, 2002, 181: $337-356[\mathrm{DOI}]$

17 Juniper S K, Tebo B M. Microbe-metal interactions and mineral deposition at hydrothermal vents. In: Karl D M, ed. The Microbiology of Deep-Sea Hydrothermal Vents. Boca Raton: CRC Press, 1995. 219-253

18 Urcuyo I A, Massothc G J, Julian D, et al. Habitat, growth and physiological ecology of a basaltic community of Ridgeia piscesae from the Juan de Fuca Ridge. Deep-Sea Res I , 2003, 50: 763-780[DOI]

19 Cary S C, Warren W, Anderson E, et al. Identification and localization of bacterial endosymbionts in hydrothermal vent taxa with symbiont-specific polymerase chain reaction amplification and in situ hybridization techniques. Mol Mar Biotechnol, 1993, 2: 51-62

20 Gaill F, Hunt S. The biology of annelid worms from high temperature hydrothermal vent regions. Rev Aquat Microbiol, 1991, 4: 107-137

21 Gaill F, Hunt S. Tubes of deep sea hydrothermal vent worms Riftia pachyptila (Vestimentifera) and Alvinella pompejana (Annelida). Mar Ecol Prog Ser, 1986, 34: 267-274[DOI]

22 Lowenstam H A. Minerals formed by organisms. Science, 1981, 211: 1126-1131[DOI]

23 Wilby P R, Briggs D E G, Riou B. Mineralization of soft-bodied invertebrates in a Jurassic metaliferous deposit. Geology, 1996, 24: $847-850[\mathrm{DOI}]$

24 van Dover C L. The Ecology of Deep-Sea Hydrothermal Vents. Princeton, New Jersey: Princeton University Press, 2000. 146-185

25 Cavanaugh C M, Gardiner C L, Jones M L, et al. Procaryotic cells in the hydrothermal vent tube worm Riftia pachyptila Jones: Possible chemoautotrophic symbionts. Science, 1981, 213: 340-342[DOI]

26 Felbeck H, Childress J J, Somero G N. Calvin-Benson cycle and sulphide oxidation enzymes in animals from sulphide-rich habitats. Nature, 1981, 293: 291-293[DOI]

27 Kelley D S, Baross J A, Delaney J R. Volcanoes, Fluids, and Life at Mid-Ocean Ridge Spreading Centers. Annu Rev Earth Planet Sci, 2002, 30: 385-491[DOI]

28 Beveridge T J, Fyfe W S. Metal oxidation by bacterial cell walls. Can J Earth Sci, 1985, 22: 1892-1898

29 Beveridge T J, Murray R G E. Sites of metal deposition in the cell wall of Bacillus subtilis. J Bacteriol, 1980, 141: 876-887

30 Ferris F G, Fyfe W S, Beveridge T J. Metallic ion binding by bacillus subtilis: Implications for the fossilization of microorganisms. Geology, 1988, 16: 149-152[DOI] 